\title{
Low-frequency noise in tunneling through a single spin
}

\author{
Y. M. Galperin,,${ }^{1,2,3}$ V. I. Kozub, ${ }^{2,3}$ and V. M. Vinokur ${ }^{3}$ \\ ${ }^{1}$ Department of Physics, University of Oslo, PO Box 1048 Blindern, 0316 Oslo, Norway \\ ${ }^{2}$ A. F. Ioffe Physico-Technical Institute of Russian Academy of Sciences, 194021 St. Petersburg, Russia \\ ${ }^{3}$ Argonne National Laboratory, 9700 S. Cass av., Argonne, IL 60439, USA
}

(Dated: November 21, 2018)

\begin{abstract}
We propose measurements of low-frequency noise in the tunneling current through a single molecule with a spin as an experimental probe for identifying a mechanism of the spin-dependent tunneling. A specific tail near the zero frequency in the noise spectrum is predicted; the amplitude and the width of being of the same order of magnitude as the recently reported peak in the noise spectrum at the spin Larmor frequency. The ratio of the spectrum amplitudes at zero- and Larmor frequencies is shown to be a convenient tool for testing theoretical predictions.

PACS numbers: 03.65.Xp, 03.65.Ta, 73.40.Gk, 73.63.Kv
\end{abstract}

Tunneling currents via a microscopic system, such as a quantum dot, or a molecule or an atom with a localized spin 1, 2, 3] attracts considerable attention in the context of the problem of quantum information processing. The tunneling current depends on spin-dynamics and thus encodes its features; at the same time, the tunneling (measuring) current influences the spin dynamics itself. Thus the tunneling via a single spin current measurement can provide information on spin orientation and its dynamics and offer an example of an indirect-continuous quantum measurement [1].

Scanning tunneling microscopy (STM) experiments [4] on a single molecule with a spin in the presence of a magnetic field $\mathbf{B}_{0}$, have revealed a peak in the current noise power spectrum (i. e., in the current autocorrelation function) $\mathcal{P}(\omega)$ at the Larmor frequency $\omega_{L}=\gamma B_{0}$, where $\gamma$ is the gyromagnetic ratio. Experiments were performed at room temperatures and found the signal-to-noise ratio $R$ (the ratio of the power at peak frequency to the shot noise power) to exceed unity and be almost independent of the orientation of the applied magnetic field $\mathbf{B}_{0}$. Experimental results [4] are hard to explain within the framework of a single-spin non-relativistic model since electrons in the leads are polarized by the same magnetic field that acts on the spin i.e. along $\mathbf{B}_{0}$, and do not couple with the oscillatory, components of the spin, which are perpendicular to $\mathbf{B}_{0}$. A possible relevance of the spin-orbit interaction has been suggested [5]. Recently, Levitov and Rashba 6] noticed that in the systems with the low space symmetry (such as a dot or a molecule near the surface) the nonvanishing orbital moment of tunneling electrons couples them to the mediating spin. They suggested that this mechanism may lead to a significant effect of the spin oscillatory component on the tunneling current.

Yet to understand experimental results [4] not only the existence of the peak in the current power spectrum is to be explained, but also the origin of the large, $R>1$, signal-to-noise ratio and its weak dependence on the orientation of $\mathbf{B}_{0}$. An important step towards formulation such a model has been done by Bulaevskii, Hruška, and
Ortiz who included in the model the non-relativistic exchange coupling of a single spin $1 / 2$ and the tunneling electrons [1, 7]. Their approach that followed [2] was based on the Keldysh formalism [8] and the Majoranafermion representation [9] for the spin, thus taking into account the nonequilibrium effects in spin dynamics explicitly. They found the spin distribution function and the current-current correlation function and discussed the dependence of $R$ and line width $\Gamma$ in the current power spectrum on the applied voltage $V$ between leads, the applied magnetic field $\mathbf{B}_{0}$, the temperature $T$. They also obtained $R$ - and $\Gamma$-dependence on the degree and orientation $\mathbf{m}_{\alpha 0}$ of electron polarization in the right- $(\alpha=$ $\mathrm{R})$ and left $(\alpha=\mathrm{L})$ current-leads in the steady state (this state establishes during the transient time after the voltage or tunneling matrix elements are switched on) 7]. The results of [7] explained several qualitative features of both average tunneling current through the spin and noise spectrum at the Larmor frequency. The quantitative agreement with the experiment was not achieved, yet the model of 7 is attractive and warrants to be explored further. The task now is to identify experimentally accessible effects that could test the underlying physics of the tunneling-through-a-spin phenomenon.

We propose measurements of the low-frequency noise (LFN) in the tunneling current as such a probe. In this Letter we develop a theory of LFN of the tunneling current adapting the Ref. 7 model. We predict a tail near zero frequency in the noise spectrum having the width of the same order of magnitude as that of the peak at $\omega_{L}$. The LFN is expressed through the same quantities as the noise at the the Larmor frequency, and thus the ratio $p=\mathcal{P}(0) / \mathcal{P}\left(\omega_{L}\right)$ turns out to be a function of the bias voltage $V$, magnetic field $\mathbf{B}_{0}$, as well as of polarization of the leads and of tunneling coupling. Thus the experimental study of behavior of the parameter $p$ offers a unique tool to check on our understanding of tunneling through a localized spin.

Low-frequency noise of the tunneling current. We use the same notations as in [7]: voltage is measured in the 
energy units, thus we write just $V$ instead of $e V$, furthermore $B$ stands for $g \mu_{B} B, T$ stands for $k_{B} T$, and $\omega$ represents $\hbar \omega$. Thus in our notations $B=\omega_{L}$. The Hamiltonian is that of the two-leads Kondo model [1, 2, 7] where the direct tunneling term is also included:

$$
\begin{aligned}
\mathcal{H}= & \mathcal{H}_{e}+\mathcal{H}_{s}+\mathcal{H}_{T}, \quad \mathcal{H}_{T}=\mathcal{H}_{\mathrm{ref}}+\mathcal{H}_{\mathrm{tr}}, \\
\mathcal{H}_{e}= & \sum_{\alpha, n, \sigma, \sigma^{\prime}}\left[\epsilon_{n \alpha} \delta_{\sigma \sigma^{\prime}}-\frac{1}{2} \mathbf{B}_{\alpha} \cdot \overrightarrow{\boldsymbol{\sigma}}_{\sigma \sigma^{\prime}}\right] c_{\alpha n \sigma}^{\dagger} c_{\alpha n \sigma^{\prime}}, \\
\mathcal{H}_{s}= & -g \mu_{B} \mathbf{B}_{0} \cdot \mathbf{S}, \\
\mathcal{H}_{\mathrm{ref}}= & \sum_{\alpha, n, n^{\prime} \sigma, \sigma^{\prime}} c_{\alpha n \sigma}^{\dagger}\left(\hat{T}_{\alpha \alpha}\right)_{\sigma \sigma^{\prime}} c_{\alpha n^{\prime} \sigma^{\prime}}, \quad \hat{T}_{\alpha \alpha}=T_{\alpha \alpha}^{(\mathrm{ex})} \mathbf{S} \cdot \overrightarrow{\boldsymbol{\sigma}}_{\sigma \sigma^{\prime}}, \\
\mathcal{H}_{\mathrm{tr}}= & \sum_{n, n^{\prime}, \sigma, \sigma^{\prime}} c_{\mathrm{R} n \sigma}^{\dagger}\left(\hat{T}_{\mathrm{RL}}\right)_{\sigma \sigma^{\prime}} c_{\mathrm{L} n^{\prime} \sigma^{\prime}}+h . c . \\
& \left(\hat{T}_{\mathrm{RL}}\right)_{\sigma \sigma^{\prime}}=T_{0} \delta_{\sigma \sigma^{\prime}}+T_{\mathrm{RL}}^{(\mathrm{ex})} \mathbf{S} \cdot \overrightarrow{\boldsymbol{\sigma}}_{\sigma \sigma^{\prime}},
\end{aligned}
$$

where $c_{\alpha n \sigma}^{\dagger}\left(c_{\alpha n \sigma}\right)$ creates (annihilates) an electron in the left or right lead (depending on $\alpha \in\{\mathrm{L}, \mathrm{R}\}$ ) in the eigenstate $n$, and with spin $\sigma$. Further, $\epsilon_{n \alpha}=\epsilon_{n}-\mu_{\alpha}$, where $\epsilon_{n}$ is the energy in the state $n$ and $\mu_{\alpha}$ is the chemical potential in the lead $\alpha$, while $\overrightarrow{\boldsymbol{\sigma}}$ represents the three Pauli matrices. $T_{\mathrm{LL}}^{(\mathrm{ex})}, T_{\mathrm{RR}}^{(\mathrm{ex})}$ and $T_{\mathrm{LR}}^{(\mathrm{ex})}$ are tunneling matrix elements due to the exchange interaction for the electron tunneling from the leads to the molecule with the spin $1 / 2$, while $T_{0}$ is the direct tunneling matrix element. We take them as real numbers. The spin localized in the molecule is described by the operator $\mathbf{S}=\left(S_{x}, S_{y}, S_{z}\right)$. Figure 1sketches the physical setup we want to study and which basically represents the model Hamiltonian $\mathcal{H}$.

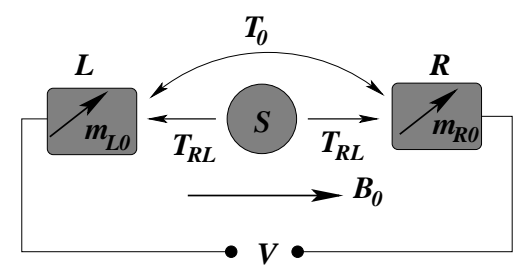

FIG. 1: Schematics of the physical system. The electronic tunneling current is established by a dc voltage $V$.

The electrical current operator can be written as

$$
\hat{I}(t)=-i e \sum_{n, n^{\prime}, \sigma, \sigma^{\prime}} c_{\mathrm{R} n \sigma}^{\dagger}(t)\left(\hat{T}_{\mathrm{RL}}\right)_{\sigma \sigma^{\prime}} c_{\mathrm{L} n^{\prime} \sigma^{\prime}}(t)+H . c .
$$

Since the spin-dependent tunneling amplitude $\hat{T}_{R L}$, Eq. (2), contains two terms, the current can be schematically presented by two vertices: $\hat{T}_{0} \equiv T_{0} \delta_{\sigma \sigma^{\prime}}$ corresponding to the spin-independent tunneling, and $\hat{T}_{s} \equiv$ $T_{\mathrm{RL}}^{(\mathrm{ex})} \mathbf{S} \cdot \boldsymbol{\sigma}_{\sigma \sigma^{\prime}}$ corresponding to the spin-dependent part.

In the following we assume $T_{\mathrm{RL}}^{(\mathrm{ex})} \ll T_{0}$. Since we are interested only in low frequencies, $\omega \ll B, V$, only $z$ component should be kept. Thus the current noise can be expressed by the schematic diagram shown in Fig. 2 The left and right electron blocks are nothing but the

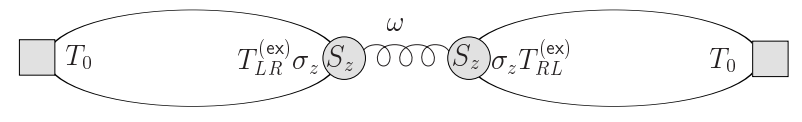

FIG. 2: Skeleton diagrams for calculation of the low-frequency noise. The wavy line corresponds to the correlation function $\left\langle\delta S_{z}^{2}\right\rangle_{\omega}$

derivatives $\partial I / \partial\left\langle S_{z}\right\rangle$, while the wavy line corresponds to the correlation function $\left\langle\delta S_{z}^{2}\right\rangle_{\omega} \equiv\left\langle\left[\delta S_{z}(t), \delta S_{z}(0)\right]_{+}\right\rangle_{\omega}$. This way we arrive at the natural expression for the lowfrequency noise,

$$
\mathcal{P}(\omega)=\left\langle[\delta I(t), \delta I(0)]_{+}\right\rangle_{\omega}=\left(\partial I / \partial\left\langle S_{z}\right\rangle\right)^{2}\left\langle\delta S_{z}^{2}\right\rangle_{\omega} .
$$

To be concrete let us restrict ourselves to the case of fully polarized electrons in the leads. Then there exists the average current proportional to the average spin, $\langle\mathbf{S}\rangle$, and given by the expression [7]

$$
\begin{aligned}
I(V) & =I_{0}(V)+\mathbf{I}_{s}(V) \cdot\langle\mathbf{S}\rangle, \\
I_{0}(V) & =\pi e\left(1+\mathbf{m}_{\mathrm{R}} \cdot \mathbf{m}_{\mathrm{L}}\right) T_{0}^{2} \rho_{0}^{2} V, \\
\mathbf{I}_{s}(V) & =2 \pi e\left(\mathbf{m}_{\mathrm{R}}+\mathbf{m}_{\mathrm{L}}\right) T_{0} T_{\mathrm{RL}}^{(\mathrm{ex})} \rho_{0}^{2} V .
\end{aligned}
$$

In the above equations $\rho_{0}$ is the density of states per spin (DOS) of the leads at the Fermi level (when leads are different $\rho_{0}^{2}=\rho_{0}^{\mathrm{L}} \rho_{0}^{\mathrm{R}}$ where $\rho_{0}^{\alpha}$ is the DOS in the lead $\alpha) ; \mathbf{m}_{\alpha}$ means the direction of electron spin polarization in the coordinate system with $z$-axis parallel to the total magnetic field, $\mathbf{B}=\mathbf{B}_{0}+\mathbf{B}_{T}$. Here $\mathbf{B}_{0}$ is the external magnetic field while $\mathbf{B}_{T}$ is the additional dc magnetic field produced by tunneling electrons.

The derivative $\partial I / \partial\left\langle S_{z}\right\rangle \equiv I_{s z}(V)$, see Eq. (5), may be found in [7], and we turn to calculation of the spin correlation function. In the equilibrium, the fluctuationdissipation theorem yields:

$$
\left\langle\delta S_{z}^{2}\right\rangle_{\omega}=\operatorname{coth} \frac{\omega}{2 T} \operatorname{Im} \chi(\omega) \approx \frac{2 \Gamma_{z}\left(1-4\left\langle S_{z}\right\rangle_{T}^{2}\right)}{\omega^{2}+\Gamma_{z}^{2}} .
$$

Here $\left\langle S_{z}\right\rangle_{T}$ is the equilibrium average spin, $\Gamma_{z}$ is the decay rate for the $S_{z}$ fluctuations.

Far from the equilibrium the fluctuation-dissipation theorem generally speaking does not apply. However, in a non-equilibrium, but yet stationary state one can still use the result of formula (8) with the appropriate expression for the average spin, $\left\langle S_{z}\right\rangle=(1 / 2) h_{f}(b)$.

$$
\left\langle\delta S_{z}^{2}\right\rangle_{\omega}=\frac{2 \Gamma_{z}\left[1-h_{f}^{2}(v, b)\right]}{\omega^{2}+\Gamma_{z}^{2}}, \quad b=\frac{B}{T}, v=\frac{V}{T} .
$$

Here $h_{f}(b, v)$ is the function calculated in [7]:

$$
\begin{aligned}
h_{f} & =-\frac{2 b\left(1-m_{\mathrm{R} z} m_{\mathrm{L} z}\right)-2 v\left(m_{\mathrm{R} z}-m_{\mathrm{L} z}\right)+b \theta_{1}}{\phi^{+}\left(1-m_{\mathrm{R} z} m_{\mathrm{L} z}\right)-\phi^{-}\left(m_{\mathrm{R} z}-m_{\mathrm{L} z}\right)+\phi(b) \theta_{1}}, \\
\theta_{1} & =\frac{T_{\mathrm{RR}}^{2}\left(1-m_{\mathrm{R} z}^{2}\right)+T_{\mathrm{LL}}^{2}\left(1-m_{\mathrm{L} z}^{2}\right)}{T_{\mathrm{RL}}^{2}}, \quad v=\frac{V}{T}, \\
\phi(b) & =b \operatorname{coth}(b / 2), \quad \phi^{ \pm} \equiv \phi(v+b) \pm \phi(v-b) . \quad(10)
\end{aligned}
$$


Equation (10) makes sense provided $m_{\mathrm{R} z} \neq 1$ and $m_{\mathrm{L} z} \neq \pm 1$. Otherwise, if $m_{\mathrm{R} z}=1, m_{\mathrm{L} z}= \pm 1$, the selfconsistency equation is an identity and the spin steady state can be any.

Equation (21) can be derived, e. g., using the the technique developed by Abrikosov 11], where a 1/2-spin was interpreted as a pseudo-Fermion with the Green function $g_{ \pm}^{(0)}(\epsilon)=(\epsilon \mp B / 2-\lambda+i \delta)^{-1}$. Here $\lambda$ is an auxiliary "chemical potential" which is send to infinity eventually. This trick allows one to remove extra unphysical states that appear because Fermi operators have more extended phase space than spin operators. The method was elaborated by Maleev for the case of dynamical defects in glasses [12]. Schematic diagrammatic representation of the correlation function $\left\langle\delta S^{2}\right\rangle_{\omega}$ is given in Fig. Bu Derivations can be carried out similarly to those in [13] where electron dephasing rates due to pseudo-spin defects were calculated. A similar procedure was also used for calculation of the energy relaxation time of the electrons in a thin wire due to magnetic impurities 14]. The correlation function is given by the diagram shown in Fig. Ba where thick dashed line corresponds to the auxiliary fermion $g_{ \pm}(\epsilon)$. An important feature of the calculation is that for calculation of $\left\langle\delta S_{z}^{2}\right\rangle_{\omega}$ one has to calculate the self energy up to the fourth order in $T_{R L}^{(\mathrm{ex})} \sigma_{x}$ as shown in Figs. 3b and 3r, see also Appendix in [12]. The wavy line, Fig. [3], corresponds to $S_{x}-S_{x}$ propagator and solid

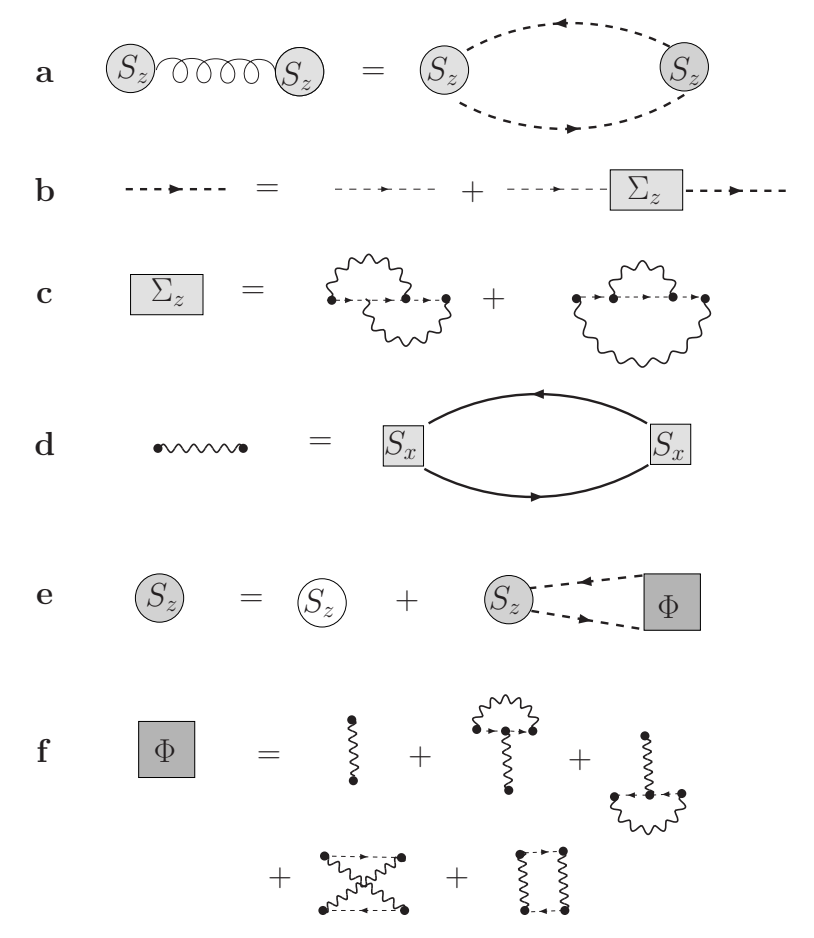

FIG. 3: Schematic diagrams for calculation of $\left\langle\delta S^{2}\right\rangle_{\omega}$. Dashed lines represent auxiliary fermions, while the vertices $S_{z}$ and $S_{x}$ represent the tunneling amplitudes $T_{R L}^{(\mathrm{ex})} \sigma_{z}$ and $T_{R L}^{(\mathrm{ex})} \sigma_{x}$, respectively. lines represent tunneling electrons. This propagator was actually calculated in [7]. A similar complication arises when calculating the $S_{z}$ (namely, $T_{R L}^{(\mathrm{ex}} \sigma_{z} S_{z}$ ) vertex part, see Fig. 33,f. When $\mathbf{m}_{\mathrm{R}}=\mathbf{m}_{\mathrm{L}}$ (except for $m_{\alpha z}= \pm 1$ ), the result is the same as for unpolarized electrons [2]:

$$
h_{f}(b, v)=\tanh \left(\frac{b}{2}\right) \frac{\phi(b)(2+\theta)}{\phi^{+}+\phi(b) \theta} .
$$

Here $\theta=\left(T_{\mathrm{LL}}^{2}+T_{\mathrm{RR}}^{2}\right) / T_{\mathrm{RL}}^{2}$. We would like to emphasize that matrix elements $T_{\alpha \alpha}$ describe electron tunneling from the lead $\alpha$ to the spin site and back while $T_{L R}$ describes spin mediated tunneling between the leads. Thus the value of $\theta$ is extremely sensitive to the location of the spin with respect to the leads. For the perfectly symmetric configuration $T_{L L}=T_{R R}=T_{L R}$, therefore $\theta=1 / 2$. Since both $T_{L L}$ and $T_{R R}$ increase dramatically with a decrease of the distance from the spin to the corresponding lead so does $\theta$ with an increase in asymmetry. As a result, in the asymmetric configurations the average spin is actually equal to its equilibrium value. This was noticed, in particular, for the similar problem of the electron tunneling mediated by the presence of the structural two-level system in [15. However for symmetric configuration the spin coupling to the electrons tunneling between the leads is as strong as its coupling to the electrons in any of the leads. As a result, the average spin is controlled by the combination of electron energy distributions within both of the leads, and it is out of equilibrium provided $v>1$. The tunneling electrons reduce the spin magnetization which drops as $1 / V$ at large $V$.

Substituting Eq. (9) into Eq. (4) we obtain

$$
\mathcal{P}_{\omega}=\frac{2 \Gamma_{z}}{\omega^{2}+\Gamma_{z}^{2}} \cdot\left(\frac{\partial I}{\partial\left\langle S_{z}\right\rangle}\right)^{2}\left[1-h_{f}^{2}(v, b)\right] .
$$

Using the expression from [7] for the spin-dependent part of the current,

$$
\partial I / \partial\langle\mathbf{S}\rangle \equiv \mathbf{I}_{s}(V)=2 \pi e\left(\mathbf{m}_{R}+\mathbf{m}_{L}\right) T_{0} T_{R L}^{(\mathrm{ex})} \rho_{0}^{2} V,
$$

we arrive at the final expression for the low-frequency noise:

$$
\begin{aligned}
& \mathcal{P}_{\omega}=P_{0} v^{2}\left[1-h_{f}^{2}(v, b)\right] \frac{2 \Gamma_{z}}{\omega^{2}+\Gamma_{z}^{2}}, \\
& P_{0}=\left[2 \pi T_{0} T_{R L}^{(\mathrm{ex})} \rho_{0}^{2} T\right]^{2}\left(m_{R z}+m_{L z}\right)^{2} .
\end{aligned}
$$

This result agrees with calculations by Shnirman et al. 10]. According to Eq. (14), the effect is strongly dependent on a degree of electron spin polarization. Here we note that the polarization can arise not only due to spin-dependent tunneling amplitude, but also as a result of electron motion through the molecule where the localized spin is located. Indeed, as it is known for semiconductor structures [16], the electrons tunneling through the barriers with no inversion center become spin-polarized if the tunneling electron has a component of wave vector $k_{\|}$parallel to the barrier plane. 
The difference between the tunneling exponents for opposite spin direction can be estimated as $\sim \gamma\left(2 m k_{\|} / \hbar^{2}\right) k d$ where $\gamma$ characterizes an efficiency of spin-orbital interaction (which for typical semiconductors is of the order of $10^{-36} \mathrm{erg} \cdot \mathrm{cm}^{3}$ ) while $d$ is the tunneling length. Of course, there is a difference between the organic molecule and a semiconductor. However the apparent absence of any pronounced symmetry in the considered situation can result in a finite $k_{\|}$, which will, in turn, lead to a spin polarization. In particular, for $k_{\|} \simeq 10^{6} \mathrm{~cm}^{-1}$ (two orders of magnitude less than typical atomic value) and for the values of $\gamma$ two order of magnitude less than the estimate given above the degree of spin polarization can be of the order of $10^{-4}$ which is larger than the spin polarization of electrons in metals at $B \sim 1 \mathrm{~T}$. Thus the factor mentioned above can significantly increase the coupling of the tunneling electrons with localized spin.

Comparison with the noise at Larmor frequency. According to Eq. (13), the low-frequency noise is represented by a Lorentzian tail with the width $\Gamma_{z}$. At $\omega \rightarrow 0$

$$
\left.\mathcal{P}_{0} \equiv \mathcal{P}_{\omega}\right|_{\omega=0}=2 P_{0} v^{2}\left[1-h_{f}^{2}(v, b)\right] \Gamma_{z}^{-1} .
$$

Let us compare this result with the maximal value of the noise at Larmor frequency, $\mathcal{P}_{L}$. According to [7], the magnitude of the noise near the Larmor frequency at $V>B$ is given by the expression

$$
\begin{aligned}
& \mathcal{P}_{\omega}=P_{1}\left[v^{2}+v b h_{f}(v, b)\right] \frac{\Gamma_{\perp}}{\Gamma_{\perp}^{2}+(\omega-B)^{2}}, \\
& P_{1}=\left(\pi T_{0} T_{\mathrm{RL}}^{\mathrm{ex}} \rho_{0}^{2} T\right)^{2}\left|\mathbf{m}_{R \perp}+\mathbf{m}_{L \perp}\right|^{2}
\end{aligned}
$$

(for fully polarized electrons). At $V<B, \mathcal{P}_{\omega}=0$.

Comparing now noise magnitudes at $\omega \rightarrow 0$ and at $\omega \rightarrow \omega_{L}$ respectively at $V>B$, we have

$$
\begin{aligned}
& \left.\mathcal{P}_{L} \equiv \mathcal{P}_{\omega}\right|_{\omega=\omega_{L}}=\left(P_{1} / \Gamma_{\perp}\right)\left[v^{2}+v b h_{f}(v, b)\right], \\
& p \equiv \frac{\mathcal{P}_{0}}{\mathcal{P}_{L}}=8 \frac{\Gamma_{\perp}}{\Gamma_{z}}\left|\frac{m_{R z}+m_{L z}}{\mathbf{m}_{R \perp}+\mathbf{m}_{L \perp}}\right|^{2} \mathcal{F}(v, b) \\
& \mathcal{F}(v, b)=\left[1-h_{f}^{2}(v, b)\right] /\left[1+(b / v) h_{f}(v, b)\right] .
\end{aligned}
$$

It rather difficult to provide realistic estimates for the ratio $\Gamma_{\perp} / \Gamma_{z}$ since spin relaxation and dephasing can be produced both by tunneling electrons and by some degrees of freedom in the leads. The contributions of the tunneling electrons to $\Gamma_{\perp}$ and $\Gamma_{z}$ are calculated in the Bloch-Redfield approximation in Ref. 10. What is important that in general $\Gamma_{\perp} \gtrsim \Gamma_{z} / 2$, and the ratio $\Gamma_{\perp} / \Gamma_{z}$ can be measured experimentally. The dimensionless function $\mathcal{F}(v, b)$ grows monotonously with $v$ for a given $b$, it tends to 1 at $v \gg b$, the plot $\mathcal{F}(v)$ shifts slightly downwards as parameter $b$ increases. Thus, the ratio $p$ can be of the order of unity.

In conclusion, we have calculated the low-frequency noise power in the tunneling current, $\mathcal{P}(0)$, and demonstrated that the ratio $p(V, T)=\mathcal{P}(0) / \mathcal{P}\left(\omega_{L}\right)$ is the universal function of dimensionless voltage and magnetic field, $e V / k_{B} T$ and $g \mu_{B} B / k_{B} T$, respectively. This opens the route for identification underlying mechanisms of the noise in tunneling current by comparison of the measured dependence of $p(V, T)$ upon voltage and temperature with the obtained $\mathcal{F}(V, T)$. We also noted that spin polarization can be obtained due to intrinsic spin polarization while tunneling through a complex molecule.

This research is supported by the US DOE Office of Science under contract No. W-31-109-ENG-38. We like to thank L. Bulaevskii and M. Hruška for useful discussions. We are specifically thankful to A. Shnirman who pointed out some mistakes in our original draft and made Ref. 10 available.

[1] L.N. Bulaevskii, G. Ortiz, Phys. Rev. Lett., 90, 040401 (2003).

[2] O. Parcollet, C. Hooley, Phys. Rev. B, 66, 085315 (2002).

[3] A.N. Korotkov, D. V. Averin, Phys. Rev. B 64, 165310 (2001); R. Ruskov, A. N. Korotkov, Phys. Rev. B, 66, 041401 (2002).

[4] Y. Manassen, R. J. Hamers, J. E. Demuth and A. J. Castellano, Jr., Phys. Rev. Lett. 62, 2531 (1989); C. Durkan, M. E. Welland, Appl. Phys. Lett. 80, 458 (2002).

[5] D. Shachal and Y. Manassen, Phys. Rev. B 44, 11528 (1991); Y. Manassen, E. Ter-Ovanesyan, D. Shachal, and S. Richter, Phys. Rev. B 48, 4887 (1993); A. V. Balatsky, I. Martin, cond-mat/0112407

[6] L. S. Levitov, E. I. Rashba, Phys. Rev. B 67, 115324 (2003).

[7] L. N. Bulaevskii, M. Hruška, and G. Ortiz, Phys. Rev. B 68, 125415 (2003).

[8] L. V. Keldysh, Sov. Phys. JETP 20,1018 (1965) [Zh. Eksp. Teor. Fiz. 47,1515 (1964)].

[9] A. M. Tsvelik, Phys. Rev. Lett. 69, 2142 (1992).

[10] A. Shnirman, D. Mozyrsky, and I. Martin (unpublished, generalization of cond-mat/0311325).

[11] A. A. Abrikosov, Physics 2 21(1965).

[12] S. V. Maleev, Sov. Phys. JETP 57, 149 (1983) [Zh. Eksp. Teor. Fiz. 84, 260 (1983)].

[13] V. V. Afonin, J. Bergli, Y. M. Galperin, V. L. Gurevich, and V. I. Kozub, Phys. Rev. B 66, 165326 (2002).

[14] G. Göppert, Y. M. Galperin, B. L. Altshuler, and H. Grabert, Phys. Rev. B 66, 195328 (2002).

[15] V. I. Kozub. Sov. Phys. - JETP 59, 1303 (1984).

[16] V. I. Perel et al., Phys. Rev. B 67, 201304(R) (2003). 